\title{
Comparison of insulin sensitivity tests across a range of glucose tolerance from normal to diabetes
}

\author{
M.P. Hermans, J. C. Levy, R. J. Morris, R. C. Turner \\ Diabetes Research Laboratories, Department of Clinical Medicine, Oxford University, Oxford, UK
}

\begin{abstract}
Aims/hypothesis. Adequate comparison of the relative performance of insulin sensitivity tests is not yet available. We compared the discrimination of four insulin sensitivity tests, commonly used in vivo, across a range of glucose tolerance.

Methods. Normal $(n=7)$, impaired glucose tolerant $(n=8)$ and Type II (non-insulin-dependent) diabetic subjects $(n=9)$ had in random order two tests from the following: frequently sampled insulin-modified intravenous glucose tolerance test (FSIVGTT-MinMod); homeostasis model assessment (HOMA) and 2-h continuous infusion of glucose with model assessment (CIGMA) with immunoreactive or specific insulin; short insulin tolerance tests (ITT). The discriminatory power of tests was assessed by the ratio of the within-subject standard deviation to the underlying between-subject standard deviation (discriminant ratio $-D R$ ). The degree to which tests measured the same variable was assessed by comparing rank correlation with the maximum expected correlation given the imprecision of the tests. The unbiased lines of equivalence taking into account the precision of tests were constructed.
\end{abstract}

Results. Reciprocal fasting plasma insulin $\left(\mathrm{FPI}^{-1}\right)$, HOMA \%S and 2-h CIGMA \% S, had similar DRs with ITT being less informative. The FSIVGTT-MinMod analysis was able to assess 13 out of 24 subjects and had a performance similar to ITT. Using specific rather than immunoreactive insulin for HOMA-CIGMA did not improve the $D R$. Reciprocal fasting plasma insulin $\mathrm{FPI}^{-1}$, $\mathrm{HOMA} \% \mathrm{~S}, 2$-h CIGMA $\% \mathrm{~S}$ and $\mathrm{S}_{\mathrm{I}}$ FSIVGTT intercorrelated more than $90 \%$ of the expected rank correlation given the imprecision of the tests, but ITT gave only limited correlation.

Conclusion/interpretation. The HOMA-CIGMA test with immunoreactive insulin provides similar information in distinguishing insulin sensitivity between subjects with normal glucose tolerance, those with impaired glucose tolerance and those with Type II diabetes as does FSIVGTT, whereas ITT is less informative. [Diabetologia (1999) 42: 678-687]

Keywords Insulin sensitivity, Type II diabetes, intravenous glucose tolerance test, insulin tolerance test, HOMA, CIGMA, reproducibility, discriminant ratio.
Received: 14 September 1998 and in revised form: 4 February 1999

Corresponding author: Dr. M.P. Hermans, Service d'Endocrinologie et Nutrition, Cliniques Universitaires St Luc, UCL 54.74, Avenue Hippocrate 54, B-1200 Brussels, Belgium Abbreviations: CIGMA, Continuous infusion of glucose with model assessment (CIGMA: 1996 update); DR, discriminant ratio; $\mathrm{FPI}^{-1}$, reciprocal of fasting plasma insulin concentration; FSIVGTT, frequently sampled intravenous glucose tolerance test; HOMA, homeostasis model assessment (HOMA: 1996 update); ITT, insulin tolerance test; $K_{g}$, slope of glucose disappearance; MinMod, Minimal Model; \% S: insulin sensitivity from HOMA or CIGMA models.
Impaired glucose tolerance (IGT) and Type II (noninsulin-dependent) diabetes mellitus result from different contributions of deficient insulin secretion and impaired insulin sensitivity [1-8]. Routine quantification of these abnormalities is rarely done as simple, user friendly methods have not been validated or received general approval $[9,10]$. The choice of therapy might depend on the causative pathophysiology in a patient and, with the prospect of insulin-sensitising drugs as well as life-style changes to improve insulin sensitivity, routine assessment of insulin sensitivity might become more appropriate than at present [11, 
12]. We have investigated different methods for measuring insulin sensitivity, to determine which is most informative across a range of glucose tolerance.

Insulin sensitivity is usually measured by assessing the relation between plasma insulin and an insulindependent metabolic variable such as plasma glucose [13-15]. The euglycaemic hyperinsulinaemic clamp is the "gold standard" for measuring insulin sensitivity $[13,16]$, but repeat plasma glucose sampling makes it both labour-intensive and costly. The widely used Minimal Model (MinMod) approach estimates insulin sensitivity by analysing glucose/insulin relations during a frequently sampled intravenous glucose tolerance test (FSIVGTT), modified with tolbutamide or exogenous insulin to obtain sufficiently high insulin concentrations to assess the glycaemic response to insulin [13-19]. Although easier to do than a clamp, it still requires approximately 30 timed-samples over a $21 / 2$-h period, and iterative determination of model variables can fail in subjects with IGT or Type II diabetes, unless insulin or tolbutamide are added [20, 21]. Doubts on its interpretation have also been raised from labelled studies [22], so that alternative approaches for routine measurement of insulin sensitivity are required.

The homeostasis model assessment (HOMA) and continuous infusion of glucose with model assessment (CIGMA) models and the short insulin tolerance tests (ITT) provide insulin sensitivity estimates that are easier to carry out [23-28]. The HOMACIGMA is a structural model of glucose/insulin interaction, with mathematical equations describing the functioning of the major effector organs. Assessment of the glucose and insulin concentrations in each person allows evaluation of the combination of deficient beta-cell function and impaired insulin sensitivity that are present. The simplest application is assessing the basal homeostasis by measuring fasting glucose and insulin concentrations with HOMA [24, 27]. Since the basal concentrations of glucose and insulin are low and require precise and sensitive assays, the alternative method evaluates the near-steady state glucose/insulin concentrations after $2 \mathrm{~h}$ of a low dose, constant glucose infusion that induces plasma glucose and insulin concentrations similar to postprandial concentrations (modelled with CIGMA) [23, 25-27]. Homeostasis model assessment and CIGMA have been validated against independent measures of insulin sensitivity and beta-cell function, including clamp-derived measures [23, 24] and are more practical, cheaper and less invasive than FSIVGTT. The short ITT is a simple direct estimate of in vivo insulin action, validated against the euglycaemic clamp, that measures the decrease in plasma glucose following an insulin bolus in the fasting state [23]. It does not estimate beta-cell function whereas the FSIVGTT, HOMA and CIGMA assess both insulin sensitivity and beta-cell function.
Methods that assess performance and agreement between tests do not provide information on their discriminatory value in clinical practice [29-33]. Validation of insulin sensitivity tests has usually consisted of correlation analysis with a reference method (usually the euglycaemic clamp), reproducibility measures and estimation of intra-subject or between-subject variation. These estimates are not combined to allow assessment of the ability of tests to discriminate in practice between subjects and to rank them for their insulin sensitivity. Assessment of the relative performance of tests requires assessment of both the reproducibility and the degree to which they are sensitive to differences within a population.

Recently a practical means of comparing imprecise tests that takes into account imprecision of tests and the degree to which they can assess differences between subjects has been developed [34]. In this study, we have assessed different insulin sensitivity tests by: firstly, measuring their ability to distinguish the insulin sensitivity of different subjects by means of their discriminant ratio; secondly, determining the degree to which different tests measure the same physiological function, using Pearson correlation coefficients adjusted for attenuation due to test imprecision and thirdly, defining unbiased lines of equivalence relating one test to another and taking into account imprecision of both tests.

Various in vivo tests of insulin sensitivity (FSIVGTT, HOMA, CIGMA, and ITT) were compared using this methodology to determine which was most efficient. This analysis was carried out over a range of subjects selected to span glucose tolerance from NGT to IGT and Type II diabetes. We also compared the respective performance of immunoreactive and specific insulin as input to estimate insulin sensitivity using HOMA and CIGMA.

\section{Subjects and methods}

Subjects. White Caucasians subjects $(n=24)$ participated in the study, which was approved by the Central Oxford Research and Ethics Committee. Glucose tolerance ranged from normoglycaemia to World Health Organisation (WHO)-defined diabetes mellitus [35]. From the subjects seven were non-diabetic with no first degree relatives with diabetes (NGT group). A further eight subjects were previously diagnosed with IGT according to an abnormal response to a $120-\mathrm{min} 75 \mathrm{~g}$ OGTT and in those subjects, as well as in NGT subjects, glucose tolerance status was confirmed at screening by an additional OGTT. A third group $(n=9)$ was diagnosed with Type II diabetes; five of them were treated with oral anti-diabetic drugs, that were stopped at least 4 weeks before the study. All subjects were on a weight maintaining diet for at least 1 month prior to testing. Subjects characteristics at recruitment are shown in Table 1 .

Over a period of 8-10 weeks, all subjects underwent in random order and in duplicate: 1) a continuous infusion of glucose with model assessment (CIGMA); 2) an insulin-modified 
Table 1. Patient characteristics

\begin{tabular}{llll}
\hline & NGT & IGT & Type II diabetes \\
\hline$n$ & 7 & 8 & 9 \\
Age (years) & $55.0 \pm 9.8$ & $65.0 \pm 8.5$ & $56.0 \pm 10.5$ \\
$\begin{array}{l}\text { Body Mass Index } \\
\left(\mathrm{kg} / \mathrm{m}^{2}\right)\end{array}$ & $24.9 \pm 3.1$ & $31.4 \pm 5.7^{\mathrm{a}}$ & $29.5 \pm 5.0$ \\
Waist-to-hip ratio & $0.81 \pm 0.08$ & $0.89 \pm 0.08^{\mathrm{a}}$ & $0.92 \pm 0.04^{\mathrm{b}}$ \\
$\begin{array}{l}\text { Body surface }\left(\mathrm{m}^{2}\right) \\
\text { HbA }_{1 \mathrm{c}}(\%)\end{array}$ & $1.73 \pm 0.23$ & $2.01 \pm 0.16^{\mathrm{a}}$ & $1.96 \pm 0.14^{\mathrm{a}}$ \\
$\begin{array}{l}\text { Fasting plasma } \\
\text { glucose (mmol/l) }\end{array}$ & $4.0 \pm 0.3$ & $5.7 \pm 0.6^{\mathrm{a}}$ & $7.7 \pm 1.2^{\mathrm{ce}}$ \\
\hline
\end{tabular}

Values are means \pm SD.

${ }^{\mathrm{a}} p<0.05,{ }^{\mathrm{b}} p<0.01$ and ${ }^{\mathrm{c}} p<0.001:$ IGT/Type II vs NGT; ${ }^{\mathrm{d}} p<0.01$ and ${ }^{\mathrm{e}} p<0.001$ : Type II vs IGT; one-way analysis of variance (ANOVA) test

FSIVGTT; 3) a short ITT combined with a homeostasis model assessment (HOMA), the ITT immediately following HOMA sampling. Tests were carried out at least 7 days apart. All volunteers were advised not to engage in unusual physical exercise for 3 days before each test. Subjects fasted from 2200 hours the previous evening. In order to minimise exercise before tests, they were advised not to exert themselves when coming to the visit (taxis were provided for the journey). Height, weight, and waist-to-hip ratio were measured on the first test day.

Methods. During each test, two antecubital cannulae, one in each arm, were inserted under local anaesthesia. The sampled arm was wrapped in electrical blankets to provide "arterialized" blood. The CIGMA test consisted of a $180 \mathrm{mg} \cdot \mathrm{min}^{-1} \cdot \mathrm{m}^{-2}$ glucose infusion for $120 \mathrm{~min}$ (infusion rate equivalent to $5 \mathrm{mg}$ / $\mathrm{kg}$ (ideal body weight)/min). The continuous low-dose infusion of glucose was started at time 0 min, with sampling at 110, 115, $120 \mathrm{~min}$ for glucose, RIA and specific insulin (2-h estimates). The reciprocal mean fasting RIA (or specific) insulin $[-10,-5$, and $0 \mathrm{~min}$; reciprocal of fasting plasma insulin concentration $\left.\left(\mathrm{FPI}^{-1}\right)\right]$ was also used as surrogate estimate for insulin sensitivity.

The modified FSIVGTT test used an intravenous glucose bolus $(0.3 \mathrm{~g} / \mathrm{kg} ; 50 \%$ solution) followed by a 5 -min insulin infusion starting $20 \mathrm{~min}$ later $(0.02 \mathrm{IU} / \mathrm{kg}$ in $4.5 \%$ albumin (Zenalb $4.5 \%$, BioProduct Laboratory, Elstree, UK)) with $3 \mathrm{~h}$ blood sampling. Following basal sampling $(-15,-10,-5$ and $0 \mathrm{~min}$ ), intravenous glucose was given at $0 \mathrm{~min}$, then sampling followed at $3,4,5,6,8,10,12,14,16$ and $19 \mathrm{~min}$; at $20 \mathrm{~min}$, insulin infusion was given, while sampling continued at $22,24,26,28,30,33,36,40,50,60,70,80,100,120,140$, 160, 180. Radioimmunoassay insulin and glucose were assayed on all samples.

For the short ITT, body weight was used to calculate the amount of intravenous insulin $(0.05 \mathrm{IU} / \mathrm{kg}$ in $4.5 \%$ albumin solution). Three fasting samples were taken at 5 min intervals immediately after insertion of the cannulae for immunoreactive (RIA) and specific insulin and glucose assays for HOMA modelling (HOMA-CIGMA version 2.1 [36]). Thereafter, bolus insulin $(0.05 \mathrm{IU} / \mathrm{kg})$ was given at time $0 \mathrm{~min}$, and sampling continued at times $1,3,5,7,9,11,13,15$ min for glucose assay. After completion of each test, subjects were provided with a light meal and advised of the possibility of reactive hypoglycaemia. After completion of the ITT, intravenous glucose ( $10 \%$ glucose solution, $300 \mathrm{ml} / \mathrm{h}$ for $30 \mathrm{~min}$ ) was also given.
Assays. Plasma glucose was measured with a hexokinase UVenzymatic method kit (Gluco-Quant Glucose, Boehringer Mannheim-BCL, Lewed, UK; assay range 0-22.2 mmol/l). Plasma immunoreactive insulin was assayed with either a double antibody RIA with ${ }^{125}$ I labelled insulin (immunoreactive insulin), with an antiserum generated in guinea pig against human insulin and a sepharose anti-guinea pig IgG generated in sheep (Pharmacia Insulin RIA 100 Kit, Pharmacia, Milton Keynes, UK; assay range 3-240 mU/1 or 22.2-1781.0 pmol/l). Specific insulin was measured with two-site sandwich ELISA. Biotinylated second antibody was detected by Streptavidin-Biotin horseradish peroxidase. Standards: Human Monocomponent Insulin with antibodies; 1) HUI-018-Murine monoclonal coating antibody and 2) OXI-Murine monoclonal, biotinylated with NHS-Biotin (Novo Nordisk Morham, Heathfield, UK and Novo Nordisk Immunochemical Department, Bagsvaerd, Denmark). Haemoglobin $\mathrm{A}_{1 \mathrm{c}}$ was measured by HPLC based on charge separation using spherical cation exchange gel with Bio-rad reagent set and DIAMAT Automated Glycosylated Haemoglobin Analyser (Bio-rad Laboratories, Hemel Hempstead, UK). Samples for RIA insulin, specific insulin and C peptide were assayed in duplicate and for glucose in singleton.

Definition and calculation of measures of insulin sensitivity. The effect of an increment in plasma insulin to enhance the fractional net disappearance of glucose from the extracellular compartment of glucose distribution is represented by $S_{I}$. The insulin sensitivity index calculated from the insulin-modified FSIVGTT using the Minimal Model approach (MinMod 2.0) $[13,17]$. Homeostasis model assessment \% $\mathrm{S}$ was assessed from fasting plasma glucose and insulin (mean of three baseline samples taken at $5 \mathrm{~min}$ intervals) and 2-h CIGMA \% S from achieved plasma glucose and insulin (mean of 3 samples at 110,115 , and $120 \mathrm{~min}$ ). Insulin tolerance test $K_{g}$, the first order rate constant for glucose disappearance rate, estimated from the slope of the regression line of $\ln$ plasma glucose compared with time (from 3 to 15 min after the insulin bolus). Reciprocal of Fasting Plasma Insulin was obtained from HOMA day values.

Comparison between tests. The methodology used for comparing different tests measuring the same underlying physiological variable was described previously [34] and covers three aspects : (1) the ability of a test to discriminate between different subjects and comparison of discrimination between different tests; (2) the underlying correlation between pairs of tests adjusting for the attenuating effect of within subject variation; and (3) in cases where the relation between a pair of tests is approximately linear, unbiased estimation of the line of equivalence between them [34].

Discrimination between subjects. For comparing $s$ tests, each measuring the same physiological variable, done $\mathrm{k}$ times on each of $n$ subjects, with a random test order, for each subject, the model is:

$\mathrm{X}_{\mathrm{ijh}}=\mu_{\mathrm{h}}+\alpha_{\mathrm{ih}}+\varepsilon_{\mathrm{ijh}} ;$ for $\mathrm{i}=1 \ldots n, \mathrm{j}=1 \ldots \mathrm{k}$ and $\mathrm{h}=1 \ldots s$

where $X_{i j h}$ is the result of the h'th test done for the $j$ 'th time on the $i^{\prime}$ th subject, $\mu_{\mathrm{h}}$ is the mean value of the variable in question on the scale of test $h$ and $\alpha_{i h}$ is the 'true' value of the i'th subject, measured on the scale of test $h$ as a deviation from the mean of that test (thus, for each test h, $\sum_{\mathrm{i}=1, \mathrm{n}} \alpha_{\mathrm{ih}}=0$ ). The $\varepsilon_{\mathrm{ijh}}$ represents day to day (biological and assay) variation, assumed to be a normally distributed random variable with mean zero, and variance $\sigma_{\mathrm{h}}^{2}$. Test results can be transformed if necessary to ensure homoscedasticity of the error term. Considering ini- 
tially a single test and dropping the h subscript, equation (1) is a one-way analysis of variance (ANOVA). In our experiments, subjects were selected to span a range of glucose tolerance and not randomly chosen and subject effects $\alpha_{i}$ are considered fixed rather than random.

Discriminant ratio. As a measure of the ability of a test to discriminate between subjects, the discriminant ratio $(D R)$ is the ratio of the underlying between-subject to the within-subject SD. Using the standard unbiased estimates of the between and within-subject variances this can be estimated by:

$D R=\sqrt{ }\left[\left(\mathrm{MS}_{\mathrm{B}}-\mathrm{MS}_{\mathrm{W}}\right) /\left(\mathrm{k} . \mathrm{MS}_{\mathrm{W}}\right)\right]$

where $\mathrm{MS}_{\mathrm{B}}$ and $\mathrm{MS}_{\mathrm{W}}$ are the between and within-subject mean squares from a standard one-way ANOVA. Equations calculating confidence limits for $D R$ s and a test for the equivalence of several $D R$ s have been derived [34].

Correlation between pairs of tests. The nature of the relation between tests can be examined by plotting the subject means for the first test against those for the second. In many cases, the relation will be approximately linear and the degree of correlation assessed using Pearson product-moment correlation coefficient $r$. In the presence of within-subject variation, the sample correlation coefficient underestimates, however, the true correlation between tests (attenuation) and the coefficient can be adjusted to give an estimate of the underlying correlation:

$r_{\text {adj }}=r /$ h

where $\mathrm{h}$ is the attenuation factor

$\mathrm{h}=\sqrt{ }\left[\left(\mathrm{DRM}_{1}^{2} /\left(1+\mathrm{DRM}_{1}^{2}\right)\right) \cdot\left(\mathrm{DRM}_{2}^{2} /\left(1+\mathrm{DRM}_{2}^{2}\right)\right)\right]$,

$D R \mathrm{M}_{\mathrm{h}}=D R_{\mathrm{h}} \cdot \sqrt{\mathrm{k}}$ is the $D R$ for the subject mean values over all days and $D R_{\mathrm{h}}$ is the $D R$ of test $\mathrm{h}, \mathrm{h}=1,2$. When the relation between tests is clearly non-linear, the Spearman rank correlation coefficient $r_{\mathrm{s}}$ should be used in place of $r$, although no universal formula exists for the attenuation of $r_{\mathrm{s}}$.

Unbiased estimation of linear relation. When the relation between a pair of tests is linear, it may be useful to obtain unbiased estimates of the gradient and intercept, although linear regression gives biased estimates because it only considers errors in the dependent variable.

The "perpendicular least squares, properly weighted" method estimates the variables of the underlying linear relation allowing for measurement error in both variables [29]. The gradient is

$\mathrm{b}=\left\{\mathrm{S}_{\mathrm{yy}}-\vartheta \cdot \mathrm{S}_{\mathrm{xx}}+\sqrt{ }\left[\left(\mathrm{S}_{\mathrm{yy}}-\vartheta \cdot \mathrm{S}_{\mathrm{xx}}\right)^{2}+4 \cdot \vartheta \cdot \mathrm{S}_{\mathrm{xy}}{ }^{2}\right]\right\} /\left(2 \cdot \mathrm{S}_{\mathrm{xy}}\right)$

where $\mathrm{S}_{\mathrm{xx}}=\Sigma_{\mathrm{i}=1, \mathrm{n}} \quad\left(\mathrm{x}_{\mathrm{i}}-\mathrm{m}_{\mathrm{x}}\right)^{2}, \quad \mathrm{~S}_{\mathrm{yy}}=\Sigma_{\mathrm{i}=1, \mathrm{n}} \quad\left(\mathrm{y}_{\mathrm{i}}-\mathrm{m}_{\mathrm{y}}\right)^{2}, \quad \mathrm{~S}_{\mathrm{xy}}=\Sigma_{\mathrm{i}=1, \mathrm{n}}$ $\left(\mathrm{x}_{\mathrm{i}}-\mathrm{m}_{\mathrm{x}}\right) \cdot\left(\mathrm{y}_{\mathrm{i}}-\mathrm{m}_{\mathrm{y}}\right), \mathrm{m}_{\mathrm{x}}$ and $\mathrm{m}_{\mathrm{y}}$ are the means of the $\mathrm{x}_{\mathrm{i}}$ and $\mathrm{y}_{\mathrm{i}}$ respectively, $\vartheta=\sigma_{\mathrm{y}}^{2} / \sigma_{\mathrm{x}}^{2}$ and $\sigma_{\mathrm{x}}$ and $\sigma_{\mathrm{y}}$ are the within-subject SD of the $x_{i}$ and $y_{i}$ respectively. The intercept is estimated as $\mathrm{a}=\mathrm{m}_{\mathrm{y}}-\mathrm{b} \cdot \mathrm{m}_{\mathrm{x}}$

Other statistical analyses. Differences between groups of subjects were assessed with either unpaired Student's $t$ test or using a one-way analysis of variance (ANOVA) and StudentNewman-Keuls multiple comparison tests, or Kruskal-Wallis non-parametric ANOVA and Dunn's multiple comparison tests. Significance was considered for $p<0.05$.

\section{Results}

The characteristics of test subjects at inclusion are given in Table 1. Body mass index was greater in IGT than in the NGT subjects, with no statistically significant difference between Type II diabetic subjects and IGT. Waist-to-hip ratio and body surface area were larger $(p<0.05)$ in subjects with Type II diabetes and IGT than in NGT subjects. Type II diabetic subjects had reduced $K_{g}$ and increased glucose both fasting and $2 \mathrm{~h}$ after CIGMA. Subjects with IGT had intermediate values, that were only different $(p<0.01)$ from NGT after CIGMA. Type II diabetic subjects had higher $(p<0.05)$ fasting insulin but following the glucose infusion this was no longer significantly different from that of normal subjects. There were no differences between groups in the glucose response to ITT (not shown).

Insulin sensitivity estimates for each group of subjects were obtained by MinMod analysis of the FSIVGTT $\left(S_{I}\right.$ FSIVGTT), HOMA and CIGMA modelling (HOMA \%S and 2-h CIGMA \%S), and by the short ITT $\left(K_{g}\right.$ ITT). Insulin sensitivity was also inferred from reciprocal fasting plasma insulin $\left(\mathrm{FPI}^{-1}\right)$. MinMod analysis of the insulin-modified FSIVGTT was convergent for both replicate tests in 13 subjects but failed to converge on one occasion in 7 subjects (2 IGT and 5 Type II diabetic) and on both occasions in 4 (1 IGT and 3 Type II diabetic). Thus, MinMod analysis of the insulin-modified FSIVGTT in 24 subjects provided repeat measurements of $S_{I}$ in all NGT, in 5/8 of IGT and in only 1/9 of Type II diabetic subjects. The "MinMod unsuccessful" group had higher $(p<0.05)$ BMI, waist-tohip ratio, fasting plasma glucose and insulin and lower $K_{g[10-20 \mathrm{~min}] \text { IVGTT }}$, HOMA $\% \mathrm{~S}, 2$-h CIGMA $\% \mathrm{~S}$ and $K_{\text {IITT. }}$. Although the insulin infusion used to modify the FSIVGTT was in the low range $(0.02 \mathrm{UI} / \mathrm{kg})$, it was sufficient to induce mild symptomatic hypoglycaemia in 2 NGT subjects.

Normal glucose tolerant subjects were more insulin sensitive than IGT or Type II diabetic subjects when assessed by $\mathrm{FPI}^{-1}$ or HOMA \% S than Type II diabetic subjects when assessed by $K_{g}$ ITT (not shown).

Difference compared with mean plots for duplicate tests (test $\mathrm{a}_{\mathrm{a}}$ vs test $_{\mathrm{b}}$ ) were heteroscedastic for HOMA, CIGMA, ITT and FPI ${ }^{-1}$, and homoscedastic after log transformation. FSIVGTT values were homoscedastic. Difference compared with mean plots on repeat test days are shown in Fig. 1. The differences observed between test replicates were largest relative to subject range for $K_{g}$ ITT and also larger with specific than RIA insulin for HOMA and 2-h CIGMA \%S. The largest day-to-day variations were found in subjects in whom $S_{I}$ could not be determined on repeat days; data plots for subjects in whom $S_{I}$ was obtainable twice $(n=13)$ are shown in Fig. 1. 


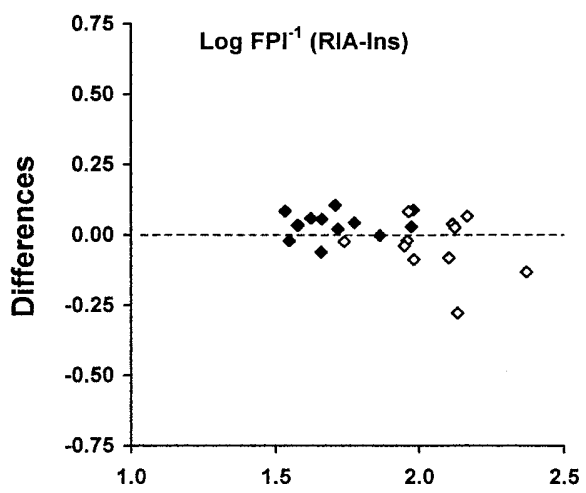

A
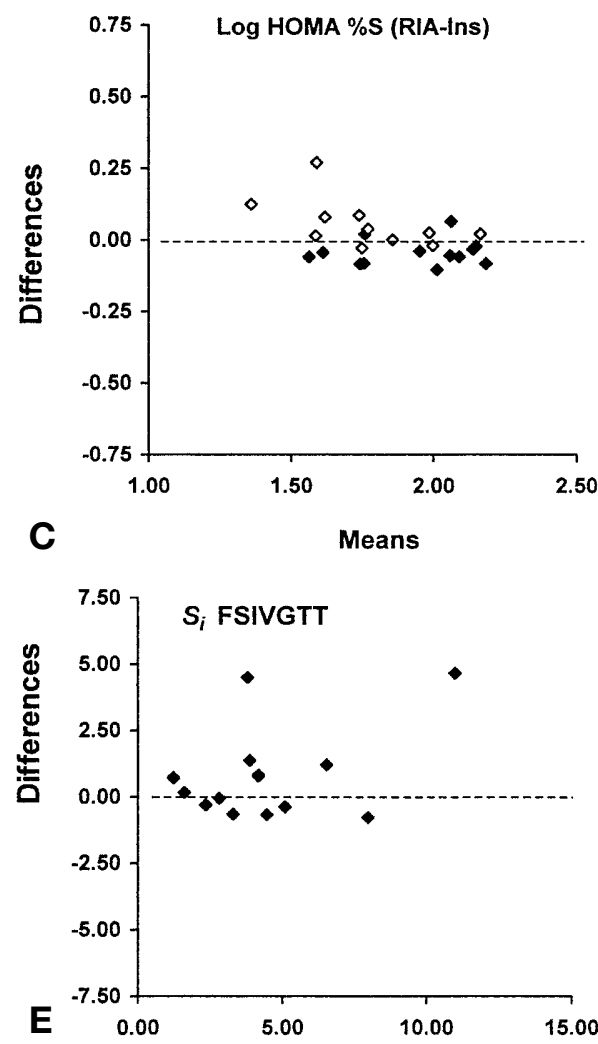

Fig. 1A-E. Means compared with differences plots showing variation across the range of glucose tolerance for five insulin sensitivity estimates measured on different days, at least 1 week apart, in 24 subjects [NGT $(n=7)$, IGT $(n=8)$ and Type II diabetes $(n=9)]$. Except for $S_{I}$ FSIVGTT, all results are log transformed: A reciprocal fasting plasma insulin concentration ( $\mathrm{FPI}^{-1}$; RIA insulin; mean of three baseline samples at $5 \mathrm{~min}$ intervals; mmol/l; upper left panel); B $K_{g}$ ITT, first order rate constant of $(\ln )$ glucose disappearance during a short insulin tolerance test (ITT, $\mathrm{min}^{-1}$; upper right panel); C HOMA \% S modelled from fasting plasma glucose and RIA insulin (mean of three baseline samples at 5 min intervals, $\%$; middle left panel); D 2-h CIGMA \% S modelled from plasma glucose and RIA insulin achieved after low-dose glucose infusion (mean of three samples at 110, 115, and $120 \mathrm{~min}, \%$; middle right panel); E $S_{I}$ FSIVGTT from MinMod $\left(10^{-4}\right.$. $\mathrm{min}^{-1} \cdot \mathrm{mU} \mathrm{l}^{-1}$ ) Open symbols identify subjects in whom $S_{I}$ FSIVGTT could not be determined on repeat days $(n=11)$ and full symbols those in whom $S_{I}$ FSIVGTT was repeatedly obtained $(n=13)$. Figures on $\mathrm{Y}$ axis omitted for clarity on right-sided graphs when values and scale identical on adjacent graphs

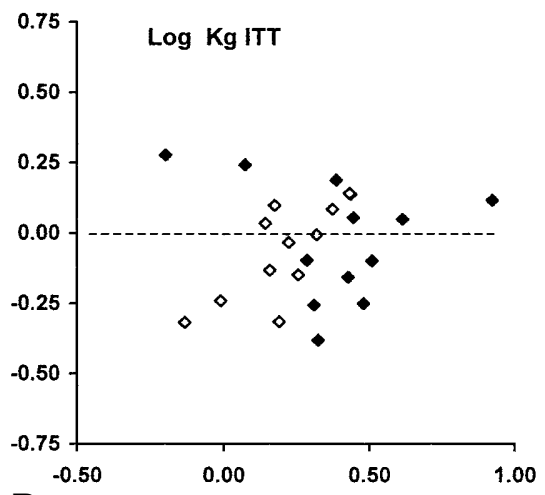

B

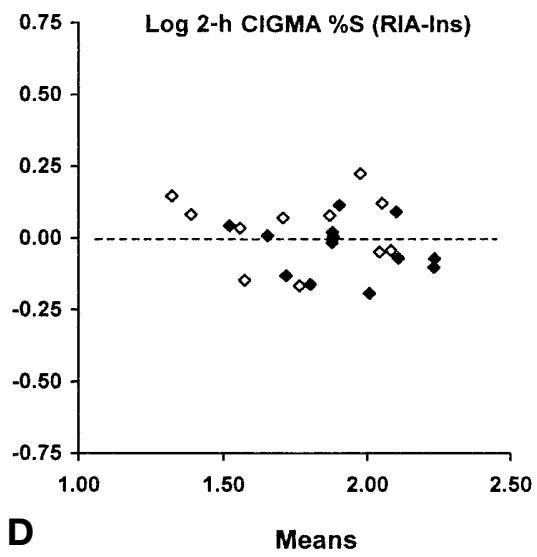

The underlying between-subject SD and global within-subject SD for each estimate of insulin sensitivity are shown on Table 2, together with the $D R$ and confidence intervals, for all subjects $(n=24)$ and for the subgroup of "FSIVGTT successful" subjects $(n=13)$. In the combined group of 24 subjects, the $D R$ of $K_{g}$ ITT was smaller than all other tests, except 2-h CIGMA \% S (specific insulin). Other tests DRs were relatively close, with 2-h CIGMA \% S (RIA insulin) having a greater $D R$ than HOMA \% S. In the "FSIVGTT successful" group $(n=13)$, absolute $D R \mathrm{~s}$ values were smaller, owing to the reduced range of individual sensitivity values resulting from exclusion of subjects with low $S_{I}$. The $D R$ of $S_{I}$ FSIVGTT was similar to that of $K_{g}$ ITT whereas 2-h CIGMA \% S (RIA insulin) had an appreciably greater $D R$ value.

Rank correlation between tests was generally high, once values were adjusted for attenuation. The measured Pearson correlation coefficients and their values adjusted for attenuation are shown on Table 3. Correlation between results of insulin sensitivity obtained in the MinMod successful group are shown as scattergrams in Fig. 2. In these subjects, $S_{I}$ correlated well with $\mathrm{FPI}^{-1}$, HOMA \% (RIA insulin) and 2-h CIGMA \% S (RIA or specific insulin), since the corrected coefficient were greater than $90 \%$ of theoretical maximum expected values when imprecision was taken into account. Correlation of $K_{g}$ ITT with other tests was not so good (Table 3). 
Table 2. Tests precision and discrimination expressed as underlying between-subject standard deviation $\left(\mathrm{SD}_{\mathrm{U}}\right)$ and as global within-subject standard deviation $\left(\mathrm{SD}_{\mathrm{W}}\right)$, and test discriminant ratio $(D R)$

\begin{tabular}{|c|c|c|c|c|c|c|}
\hline & $\mathrm{SD}_{\mathrm{U}}$ & $\mathrm{SD}_{\mathrm{W}}$ & $\mathrm{SD}_{\mathrm{U}}$ & $\mathrm{SD}_{\mathrm{W}}$ & $D R$ & \\
\hline$n$ & 24 & & 13 & & 24 & 13 \\
\hline$S_{1}$ FSIVGTT & - & - & 0.25 & 0.14 & - & $1.77(1.52-2.06)$ \\
\hline Log $\mathrm{FPI}^{-1}$ (RIA insulin) & 0.22 & 0.08 & 0.15 & 0.07 & $2.92^{\mathrm{a}}(2.84-3.01)$ & $2.08(1.96-2.21)$ \\
\hline $\begin{array}{l}\text { Log HOMA \% S } \\
\text { RIA insulin } \\
\text { specific insulin }\end{array}$ & $\begin{array}{l}0.23 \\
0.23\end{array}$ & $\begin{array}{l}0.07 \\
0.08\end{array}$ & $\begin{array}{l}0.14 \\
0.17\end{array}$ & $\begin{array}{l}0.07 \\
0.07\end{array}$ & $\begin{array}{l}3.04^{\mathrm{a}}(2.95-3.13) \\
3.01^{\mathrm{a}}(2.82-3.21)\end{array}$ & $\begin{array}{l}2.08(1.96-2.21) \\
2.42(2.12-2.76)\end{array}$ \\
\hline
\end{tabular}

Values are from individual tests and means of their duplicates in the combined groups $(n=24 ; 7$ NGT, 8 IGT and 9 Type II diabetic subjects); for comparison of FSIVGTT with other estimates, $n=13$ subjects [ $n=5$ (IGT) and 1 (Type II diabetes] respectively, i.e. subjects with duplicates convergent MinMod].

Except for $S_{1}$ FSIVGTT, all results log transformed for homoscedasticity. Confidence intervals for $D R \mathrm{~s}(2.5-97.5 \%)$ in parentheses. ${ }^{\mathrm{a}}$ : $p<0.05$ and ${ }^{\mathrm{b}} p<0.02$ for unequality of $D R$ with $\log K_{\mathrm{g} \text { ITT }}$ at a significance of 0.05 for a statistic exceeding the $95^{\text {th }}$ centile of a $C h i^{2}$ distribution

Table 3. Pearson correlation coefficients measured between tests with values adjusted for attenuation in parentheses

\begin{tabular}{|c|c|c|c|c|c|c|}
\hline & \multirow{2}{*}{$\begin{array}{l}\mathrm{S}_{1} \text { FSIVGTT } \\
n=13\end{array}$} & \multirow{2}{*}{$\begin{array}{l}\text { Log } \mathrm{FPI}^{-1} \\
\text { RIA insulin }\end{array}$} & \multirow[t]{2}{*}{$\log K_{\mathrm{g}}$ ITT } & \multicolumn{2}{|c|}{ Log HOMA \% S } & \multirow{2}{*}{$\begin{array}{l}\text { Log CIGMA \% S } \\
\text { RIA insulin }\end{array}$} \\
\hline & & & & $\begin{array}{l}\text { RIA insulin } \\
n=24\end{array}$ & specific insulin & \\
\hline \multicolumn{7}{|l|}{$\log _{\mathrm{FPI}^{-1}}$} \\
\hline RIA insulin & $0.88(1.00)$ & & & & & \\
\hline $\log K_{\mathrm{g}}$ ITT & $0.60(0.69)$ & $0.61(0.68)$ & & & & \\
\hline \multicolumn{7}{|l|}{ Log HOMA \% S } \\
\hline RIA insulin & $0.88(1.00)$ & $0.92(0.97)$ & $0.68(0.76)$ & & & \\
\hline specific insulin & $0.76(0.84)$ & $0.90(0.95)$ & $0.68(0.76)$ & $0.91(0.96)$ & & \\
\hline \multicolumn{7}{|l|}{ Log CIGMA \% S } \\
\hline RIA insulin & $0.86(0.93)$ & $0.83(0.87)$ & $0.50(0.56)$ & $0.79(0.83)$ & $0.71(0.75)$ & \\
\hline specific insulin & $0.88(1.00)$ & $0.88(0.94)$ & $0.51(0.57)$ & $0.84(0.88)$ & $0.76(0.81)$ & $0.94(1.00)$ \\
\hline
\end{tabular}

All correlations were calculated from means of tests duplicates (from log results) in $n=24$ subjects (7 NGT, 8 IGT and 9 with Type II diabetes) except for correlations between FSIVGTTMinMod ( $n=13$ subjects). For correlation between $\mathrm{FPI}^{-1}$ or

The $\alpha$ and $\beta$ coefficients of equations expressing the lines of "true" equivalence relating two given tests are described in Table 4 . These coefficients are provided for all combinations of estimates of insulin sensitivity and enable construction of "true" lines of equivalence $(\mathrm{y}=\alpha+\beta \mathrm{x}$, i. e. the lines between physiologically equivalent points of insulin sensitivity measured by two different methods) for any pair of tests, taking into account random variation in both variables measured. These slopes and intercepts are unbiased and independent of $\mathrm{V}_{\mathrm{w}}$.

\section{Discussion}

This study was undertaken to compare the discriminating performance in a series of in vivo insulin sensitivity tests. Our data show that cheap, simple and non-invasive insulin sensitivity indices such as $\mathrm{FPI}^{-1}$
HOMA \% S (RIA insulin) and other estimates, fasting glucose and insulin concentrations were obtained from the mean of tests done on different days

and HOMA or 2-h CIGMA modelling of $\% \mathrm{~S}$ were as able to discriminate differences between subjects as the more widely used FSIVGTT-MinMod or better than the more invasive ITT. We simultaneously compared different tests for intra-subject variability, discrimination, correlation and "lines of equivalence". The ability of a test to distinguish individual subjects has been assessed by its discriminant ratio $(D R)$ and the relation between tests has been expressed as Pearson correlation coefficients adjusted for the attenuating effect of within-subject variation. In addition, we present $\alpha$ and $\beta$ coefficients for the equations of the lines of equivalence between pairs of tests.

Reciprocal fasting plasma insulin, HOMA \% $\mathrm{S}$ or 2-h CIGMA \% $\mathrm{S}$ achieved as high a $D R$ as did the more intensive insulin sensitivity tests, FSIVGTT and ITT. Apart from the ITT, these tests showed high intercorrelation coefficients, and good correla- 

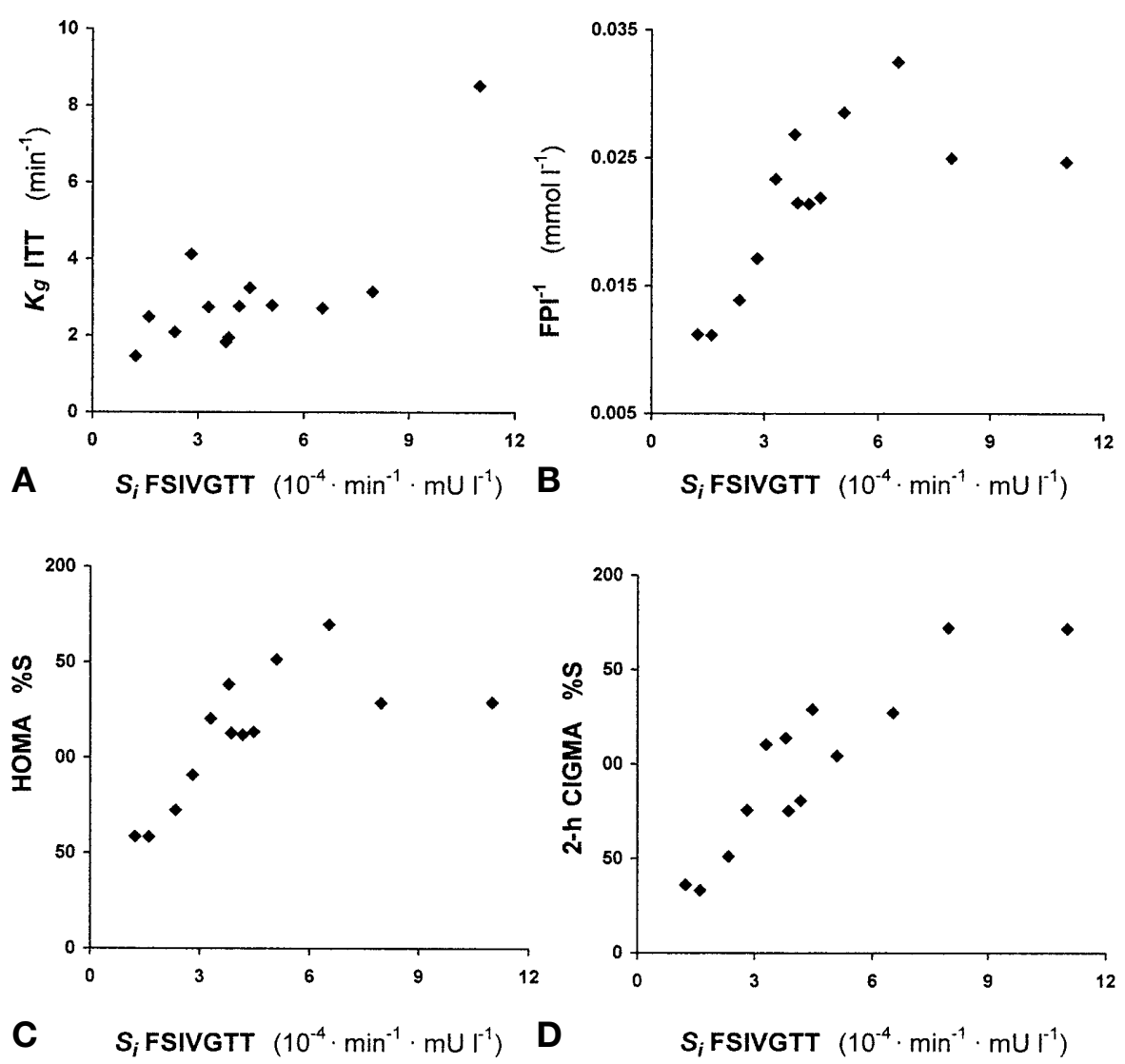

Fig. 2 A-D. Correlation between the means of two $S_{I}$ FSIVGTT-MinMod measurements and the means of two $\left(K_{g}\right.$ ITT, CIGMA \% S) or six determinations ( $\left.\mathrm{FPI}^{-1}, \mathrm{HOMA} \% \mathrm{~S}\right)$ of other estimates of insulin sensitivity: A $K_{g}$ ITT and B reciprocal fasting plasma RIA-insulin $\left(\mathrm{FPI}^{-1}\right.$, mean of three baseline samples, upper panel), and C HOMA \% S (RIA insulin) and D 2-h CIGMA \% S (RIA insulin; lower panel). Results from $n=13$ subjects (seven NGT, five IGT and one Type II diabetic)

tion with $S_{I}$. The 2-h CIGMA \% S (RIA insulin) had the best performance but additional studies would be needed to confirm whether this is so. This test is easier to do than the FSIVGTT and also provides

measures of beta-cell function and glucose tolerance $[23,25]$. The less precise results with CIGMA using specific rather than RIA insulin show how the results are critical on assay methods. The short insulin tolerance test performance was less than all other tests, due to poor reproducibility. The performance of FSIVGTT-MinMod $S_{I}$ was similar to that of $\mathrm{FPI}^{-1}$, HOMA and 2-h CIGMA \% S.

With the amount of insulin used in our study, it was expected from all the previously published data that FSIVGTT-MinMod would be more successful for ascertaining insulin sensitivity in the less hyperglycaemic and insulin resistant subjects. It is possible that extending the sampling period for longer, or addition

Table 4. Intercept (alpha) and slope (beta) coefficients of the unbiased lines of equivalence equations relating pairs of insulin sensitivity tests

\begin{tabular}{|c|c|c|c|c|c|c|c|c|c|c|c|c|c|}
\hline \multirow[t]{2}{*}{ Coefficients } & & \multicolumn{2}{|c|}{$S_{1}$ FSIVGTT } & \multicolumn{2}{|c|}{$\log K_{\mathrm{g}} \mathrm{ITT}$} & \multicolumn{2}{|c|}{$\begin{array}{l}\text { Log } \\
\text { FPI }^{-1} \\
\text { RIA insulin }\end{array}$} & \multicolumn{2}{|c|}{$\begin{array}{l}\text { Log } \\
\text { HOMA \%S } \\
\text { RIA insulin }\end{array}$} & \multicolumn{2}{|c|}{$\begin{array}{l}\text { Log } \\
\text { HOMA \% S } \\
\text { specific insulin }\end{array}$} & \multicolumn{2}{|c|}{$\begin{array}{l}\text { Log 2-h } \\
\text { CIGMA \% S } \\
\text { RIA insulin }\end{array}$} \\
\hline & & alpha & beta & alpha & beta & alpha & beta & alpha & beta & alpha & beta & alpha & beta \\
\hline $\log K_{g}$ ITT & & -2.72 & 0.71 & & & & & & & & & & \\
\hline Log $\mathrm{FPI}^{-1}$ & RIA insulin & -4.31 & 0.58 & -2.16 & 1.02 & & & & & & & & \\
\hline Log HOMA \% S & RIA insulin & -0.58 & 0.58 & 1.55 & 1.03 & 3.73 & 1.00 & & & & & & \\
\hline Log HOMA \% S & specific insulin & -1.12 & 0.70 & 1.52 & 1.05 & 3.74 & 1.03 & -0.07 & 1.02 & & & & \\
\hline
\end{tabular}

All values calculated from means of tests duplicates (from $\log$ results) in $n=24$ subjects (7 NGT, 8 IGT and 9 with Type II diabetes) except for correlations between FSIVGTT-MinMod ( $n=13$ subjects) 
of an artificial point at 240 or 360 min could have decreased the failure rate in IGT and Type II diabetic subjects. The FSIVGTT is often considered a more feasible alternative to the euglycaemic clamp against which it has been validated [18]. It is, however, less efficient than some other tests that provide data across a greater range of patients $[20,21]$. We used a bolus insulin dose of $0.02 \mathrm{IU} / \mathrm{kg}$ in the insulin-modified FSIVGTT as recommended [38] and similar to that used in another study in which it was shown that this dose is a good match to peripheral plasma glucose and insulin responses from a tolbutamide-modified FSIVGTT [39]. Although it is possible that a larger insulin bolus could make the test more applicable in insulin resistant and diabetic patients, the dose we used was sufficient to induce hypoglycaemia in some normal subjects.

We found a rate of convergence failure with bolus insulin of $0.02 \mathrm{IU} / \mathrm{kg}$ of about $38 \%$ (IGT) and about $90 \%$ (Type II diabetes), and the "failure" group were predominantly those with low insulin sensitivity values on other tests, this was not unexpected since they had IGT or diabetes. A $50 \%$ failure of a 22 -samples or 12-samples FSIVGTT (modified with 0.03 IU/ $\mathrm{kg}$ insulin) to provide insulin sensitivity in non-diabetic, mostly obese, subjects has been reported [20]. A larger dose (0.03-0.05 IU $/ \mathrm{kg})$ may have provided more estimates of insulin sensitivity, but would probably produce hypoglycaemia in more subjects. Although it might seem reasonable to vary the dose according to the person being studied, this is likely to produce systematic differences in the resulting measure of insulin sensitivity. Our data suggest that exogenous insulin should be used at a low $(0.02 \mathrm{IU} / \mathrm{kg})$ dose when a FSIVGTT is contemplated in NGT and IGT subjects with previously unknown insulin sensitivity.

The repeatability of tolbutamide-modified FSIVGTT-MinMod has been reported [40] as similar to that of a clamp in NGT subjects (CV 14\%), although in another report [41] the CV was $22 \%$ and we found a CV of about $30 \%$. Procedures with fewer samples may be applicable in NGT subjects [42, 43], although it has been found [44] that reduced sampling gives less precision, with CVs (for $n$ samples) of 18 (30), 29 (12), and $27 \%$ (13). It is doubtful whether reduced sampling would be effective in subjects with IGT or diabetes who can fail to converge with results from 31 samples.

Studies on reproducibility for CIGMA estimates show that the inter-assay CV of plasma insulin measurement is a major component of imprecision [23, 27]. This was shown in the present study, in which use of a less precise, but presumably more accurate, specific insulin assay gave less good discrimination. Homeostasis Model Assessment evaluates fasting glucose and insulin measurements and requires precise and sensitive assays. We took three fasting sam- ples over $10 \mathrm{~min}$ to improve precision and to take into account the $15 \mathrm{~min}$ pulsatility of insulin secretion in normal subjects [45]. Although we found HOMA gave similar $D R$ s to 2-h CIGMA, if less precise glucose and insulin measurements were available CIGMA might be relatively more applicable than the present study indicates.

Our data suggest that in NGT and IGT categories, no single test is definitely superior to another, although the 2-h CIGMA \% S (RIA insulin) is perhaps more informative. This test is easier to carry out than the FSIVGTT and also gives a measure of betacell function and of glucose tolerance. The relative superiority of $\mathrm{FPI}^{-1}$, HOMA \% S and CIGMA \% S compared with FSIVGTT-MinMod is somewhat arguable in subjects in which fits were obtained with the Minimal Model.

A simple method for quantification of insulin sensitivity from an IVGTT $\left(K_{g} / \Delta_{\text {incremental }} \mathrm{IRI}_{0-40 \text { min }}\right)$ has been reported [46]. This alternative analysis could be of value in conditions where traditional reference procedures (clamp or FSIVGTT-MinMod) are not feasible. We could not compare its performance in this study, as exogenous insulin during the FSIVGTT rendered impossible the determination of incremental endogenous insulin beyond the 20th min.

The short ITT is reported to have good precision (at an insulin dose of $0.1 \mathrm{UI} / \mathrm{kg}$ ), with a CV of $6 \%$ in obese NGT subjects [47] and $9 \%$ in normal subjects [28], whereas in non-obese NGT subjects and using a $0.05 \mathrm{UI} / \mathrm{kg}$ insulin bolus within-subject CVs of $13 \%$ has been reported [48]. With this dose we found CVs of about $30 \%$ in our group spanning the range of glucose tolerance. It is possible that the insulin dose might have contributed to the discrepancy, although it was sufficient to induce hypoglycaemia in some subjects.

Reciprocal of fasting plasma insulin concentration alone performed well as a surrogate estimate of insulin sensitivity, both in terms of $D R$ and of correlation with other tests. This is in accordance with a previous report [49] in which a correlation between FPI and resistance to insulin-mediated glucose uptake in normal and diabetic subjects during an insulin suppression test was described and also a negative correlation with insulin sensitivity when the latter was less than $3 \mathrm{~min}^{-1} / \mu \mathrm{U} \mathrm{ml}^{-1}$ (MinMod analysis) in lean and obese non-diabetic subjects [18]. A correlation $(p<0.001)$ between fasting plasma insulin and insulin sensitivity was also described in IGT subjects [50]. A non-linear relation with FPI using FSIVGTT-MinMod in non-diabetic subjects with varying degrees of obesity has been described [51] and a negative correlation was also reported during euglycaemic clamps. Since FPI is increased when impaired insulin sensitivity is present, secondary to the "resistance-induced" increment in plasma glucose, these associations would be expected. The HOMA model shows that re- 
duction of FPI from the error signal in the homeostatic feed-back loops introduced by deficient insulin secretion is small, which explains why $\mathrm{FPI}^{-1}$ alone is a reasonable estimator of insulin sensitivity. Nevertheless, the addition of measurement of fasting plasma glucose allows for the error signal and, in theory, HOMA provides a more accurate measure of insulin sensitivity, alongside a beta-cell function estimate.

It has been suggested [9] that the ideal method for measuring insulin sensitivity should satisfy five requirements: 1) to achieve insulin concentrations high enough to stimulate glucose metabolism and detect small differences in sensitivity of glucose uptake to insulin; 2) to distinguish between peripheral and hepatic insulin sensitivity; 3) to measure steady-state conditions; 4) to rest on physiologically sound assumptions about body glucose system and 5) to achieve a degree of hyperglycaemia not overtly nonphysiological. Although no such perfect test has yet been proposed for routine use, HOMA fulfils three criteria and 2-h CIGMA four criteria. A candidate test should score high in analysis of test performance and be simple, safe and cheap. Two large studies illustrate the application of HOMA for assessing for the natural course of diabetes [52] or the response to pharmacological intervention [11].

In conclusion, simple non-invasive estimates can be used to discriminate subjects for their insulin sensitivity. Continuous infusion of glucose with model assessment appears to be a better discriminatory test in precision and agreement analysis. The HOMACIGMA models, using RIA insulin, are candidates for routine measurement of insulin sensitivity. Using specific insulin as input offered no direct advantage over RIA insulin, although this may change when more precise specific assays are used. Different studies have used different methods of assessing insulin sensitivity and the methodology used here allows for unbiased lines of equivalence to be established between tests, a practical means of comparing results between studies, although allowance needs to be made when different procedures or assays have been used.

Acknowledgements. The study was done with the aid of grants from the Royal Society and Oxford District Health Services Research Committees. We are grateful to all volunteer subjects, for the advice from Dr. S. Coppack, to Ms. P. Sutton, Mr. R. Mair and Dr. S. Manley for the assays, to Ms. B. Barrow and Ms. N. Walravens for assistance with the tests. The MinMod program was kindly provided by Professor R. N. Bergman. Dr. M.P. Hermans was on leave from the Catholic University of Louvain Scientific Development Trust (Fonds du Développement Scientifique de l'Université Catholique de Louvain) and granted a Fellowship from the Belgian Diabetic Association (Fonds Suzanne and Jean Pirart).

\section{References}

1. Ward WK, Johnston CLW, Beard JC, Benedetti TJ, Halter JB, Porte D Jr (1985) Insulin resistance and impaired insulin secretion in subjects with histories of gestational diabetes mellitus. Diabetes 34: 861-869

2. UK Prospective Diabetes Study V (1988) Characteristics of newly presenting type 2 diabetic patients: estimated insulin sensitivity and islet B-cell function. Diabet Med 5: 444-448

3. DeFronzo RA (1988) The triumvirate: $\beta$-cell, muscle, liver. A collusion responsible for NIDDM. Diabetes 37: 667-687

4. Erikson J, Franssila-Kallunki A, Ekstrand A et al. (1989) Early metabolic abnormalities in persons at increased risk for non-insulin-dependent diabetes mellitus. N Eng J Med 321: 337-343

5. Page RCL, Walravens EKN, Levy JC, Stratton IM, Turner RC (1992) Prevalence and pathophysiology of impaired glucose tolerance in three different high-risk groups. Metabolism 42: 932-938

6. Beck-Nielsen H, Groop L (1994) Metabolic and genetic characterization of prediabetic states. Sequence of events leading to non-insulin-dependent diabetes mellitus. J Clin Invest 94: 1714-1721

7. Zawalich WS, Kelley GG (1995) The pathogenesis of NIDDM: the role of the pancreatic beta-cell. Diabetologia 38: 986-991

8. Reaven GM (1995) The fourth musketeer - from Alexandre Dumas to Claude Bernard. Diabetologia 38: 3-13

9. Groop LC (1993) Insulin resistance and insulin deficiency in the pathogenesis of Type 2 (non-insulin-dependent) diabetes mellitus: errors of metabolism or of methods? Diabetologia 36: 1326-1331

10. Taylor SI, Accili D, Imai Y (1994) Insulin resistance or insulin deficiency. Which is the primary cause of NIDDM. Diabetes 43: 735-740

11. Kumar S, Boulton AJM, Beck-Nielsen $\mathrm{H}$ et al. for the Troglitazone study group (1996) Troglitazone, an insulin action enhancer, improves metabolic control in NIDDM patients. Diabetologia 39: 701-709

12. Henry RR (1996) Effect of troglitazone on insulin sensitivity. Diabet Med 13: S148-S150

13. Bergman RN, Finegood D, Ader M (1985) Assessment of insulin sensitivity in vivo. Endocrine Reviews 6: 45-86

14. Ader M, Bergman RN (1987) Insulin sensitivity in the intact organism. In: Baillières Clin Endocrinol Metab 1: 879-910

15. Bergman RN, Hope ID, Yang YJ et al. (1989) Assessment of insulin sensitivity in vivo: a critical review. Diabetes Metab Rev 5: 411-429

16. DeFronzo RA, Tobin JD, Andres R (1979) Glucose clamp technique: a method for quantifying insulin secretion and resistance. Am J Physiol 237:E214-E223

17. Pacini G, Bergman RN (1986) MINMOD: a computer program to calculate insulin sensitivity and pancreatic responsivity from the frequently sampled intravenous glucose tolerance test. Comput Methods Programs Biomed 23: 113-122

18. Bergman RN, Prager R, Volund A, Olefsky JM (1987) Equivalence of the insulin sensitivity index in man derived from the Minimal Model method and the euglycemic glucose clamp. J Clin Invest 79: 790-800

19. Bergman RN (1989) Toward physiological understanding of glucose tolerance. Minimal-model approach. Diabetes 38: $1512-1527$

20. Saad MF, Anderson RL, Laws A et al. for the Insulin Resistance Atherosclerosis Study (1994) A comparison between the Minimal Model and the glucose clamp in the as- 
sessment of insulin sensitivity across the spectrum of glucose tolerance. Diabetes 43: 1114-1121

21. Anderson RL, Hamman RF, Savage PJ et al. for the Insulin Resistance Atherosclerosis Study (1995) Exploration of simple insulin sensitivity measures derived from frequently sampled intravenous glucose tolerance (FSIVGT) tests. Am J Epidemiol 142: 724-732

22. Caumo A, Giacca A, Morgese M, Pozza G, Micossi P, Cobelli C (1991) Minimal Model of glucose disappearance: lessons from the labelled IVGTT. Diabet Med 8: 822-832

23. Bonora E, Moghetti P, Zancanaro C et al. (1989) Estimates of in vivo insulin action in man: comparison of insulin tolerance tests with euglycemic and hyperglycemic glucose clamp studies. J Clin Endocrinol Metab 68: 374-378

24. Hosker JP, Matthews DR, Rudenski AS et al. (1985) Continuous infusion of glucose with model assessment: measurement of insulin resistance and $\beta$-cell function in man. Diabetologia 28: 401-411

25. Matthews DR, Hosker JP, Rudenski AS, Naylor BA, Treacher DF, Turner RC (1985) Homeostasis Model Assessment: insulin resistance and $\beta$-cell function from fasting plasma glucose and insulin concentration in man. Diabetologia 28: 412-419

26. Levy JC, Rudenski AS, Burnett M, Knight R, Matthews DR, Turner RC (1991) Simple empirical assessment of $\beta$ cell function by a constant infusion of glucose test in normal and Type 2 (non-insulin-dependent) diabetic subjects. Diabetologia 34: 488-499

27. Rudenski AS, Matthews DR, Levy JC, Turner RC (1991) Understanding 'insulin resistance': both glucose resistance and insulin resistance are required to model human diabetes. Metabolism 40: 908-917

28. Turner RC, Levy JC, Rudenski AS, Hammersley M, Page $\mathrm{R}$ (1993) Measurement of insulin resistance and beta-cell function: the HOMA and CIGMA approach. In: Belfiore F, Bergman RN, Molinatti GM (eds) Current topics in diabetes research. Karger, Basel, 12: 66-75

29. Riggs DS, Guarnieri JA, Addelman S (1978) Fitting straight lines when both variables are subject to error. Life Sciences 22: 1305-1360

30. British Standards Institution (1979) Precision of test methods I: Guide for the determination and reproducibility for a standard test method (BS 5497, part 1), British Standards Institution, London

31. Godfrey K (1985) Simple linear regression in medical research. N Eng J Med 313: 1629-1636

32. Bland JM, Altman DG (1986) Statistical methods for assessing agreement between two methods of clinical measurement. Lancet i: $307-310$

33. Spilker B (1991) Validation of clinical tests and measures. In: Guide to clinical trials. Raven Press, New York, pp 313-319

34. Levy JC, Morris RJ, Hammersley M, Turner RC (1999) Comparison of imprecise physiological tests: discrimination, maximum expected rank correlation and unbiased equivalence. Am J Phys (in press)

35. World Health Organisation Study Group on Diabetes Mellitus (1985) Technical Report Series No 727, WHO, Geneva

36. Levy JC, Matthews DR, Hermans MP (1998) Correct Homeostasis Model Assessment (HOMA) evaluation uses the computer program. Diabetes Care 21: 2191-2192
37. Raggat PR (1989) Duplicates or singletons? An analysis of the need for replication in immunoassay and a computer program to calculate the distribution of outliers, error rate and the precision profile from assay duplicates. Ann Clin Biochem 26: 23-37

38. Taniguchi A, Nakai Y, Fukushima M et al. (1992) Pathogenic factors responsible for glucose intolerance in patients with NIDDM. Diabetes 41: 1540-1546

39. Finegood DT, Hramiak IM, Dupré J (1990) A modified protocol for estimation of insulin sensitivity with the Minimal Model of glucose kinetics in patients with insulin-dependent diabetes. J Clin Endocrinol Metab 70: 1538-1549

40. Ferrari P, Alleman Y, Shaw S, Riesen W, Weidmann P (1991) Reproducibility of insulin sensitivity measured by the minimal model method. Diabetologia 34: 527-530

41. Avogaro A, Bristow JD, Bier DM, Cobelli C, Toffolo G (1989) Stable-label intravenous glucose tolerance test Minimal Model. Diabetes 38: 1048-1055

42. Steil GM, Volund A, Kahn SE, Bergman RN (1993) Reduced sample number for calculation of insulin sensitivity and glucose effectiveness from the Minimal Model. Suitability for use in population studies. Diabetes 42: 250-256

43. Duysinx BC, Scheen AJ, Gerard PL, Letiexhe MR, Paquot N, Lefebvre PJ (1994) Measurement of insulin sensitivity by the minimal model method using a simplified intravenous glucose tolerance test: validity and reproducibility. Diabetes Metab 20: 425-432

44. Coates PA, Ollerton RL, Luzio SD, Ismail IS, Owens DR (1993) Reduced sampling protocols in estimation of insulin sensitivity and glucose effectiveness using the Minimal Model in NIDDM. Diabetes 42: 1635-1641

45. Matthews DR, Lang DA, Burnett MA, Turner RC (1983) Control of pulsatile insulin secretion in man. Diabetologia 24: 231-237

46. Galvin P, Ward G, Walters J et al. (1992) A simple method for quantitation of insulin sensitivity and insulin release from an intravenous glucose tolerance test. Diabet Med 9: 921-928

47. Akinmokun A, Selby PL, Ramaiya K, Alberti KGM (1992) The short insulin tolerance test for determination of insulin sensitivity: a comparison with the euglycaemic clamp. Diabet Med 9: 432-437

48. Hirst S, Phillips DIW, Vines SK, Clark PM, Hales CN (1993) Reproducibility of the short insulin tolerance test. Diabet Med 10: 839-842

49. Olefsky JM, Farquhar JW, Reaven G (1973) Relationship between fasting plasma insulin level and resistance to insulin-mediated glucose uptake in normal and diabetic subjects. Diabetes 22: 507-513

50. Bonora E, Manicardi V, Zavaroni I, Coscelli C, Butturini U (1987) Relationships between insulin secretion, insulin metabolism and insulin resistance in mild glucose intolerance. Diabete Metabolisme 13: 116-121

51. Kahn SE, Prigeon RL, McCullogh DK et al. (1993) Quantification of the relationship between insulin sensitivity and $\beta$-cell function in human subjects. Evidence for a hyperbolic function. Diabetes 42: 1663-1672

52. Haffner SM, Gonzales C, Miettinen H, Kennedy E, Stern MP (1996) A prospective analysis of the HOMA model. The Mexico City Diabetes Study. Diabetes Care 19: 1138-1141 